\title{
Continuous Selections of Lipschitz Extensions in Metric Spaces
}

\author{
Rafa Espínola ${ }^{a}$, Adriana Nicolae $e^{b, c}$ \\ ${ }^{a}$ Departamento de Análisis Matemático - IMUS, Universidad de Sevilla, Apdo. de Correos 1160, 41080 Sevilla, Spain \\ ${ }^{b}$ Department of Mathematics, Babeş-Bolyai University, Kogălniceanu 1, 400084 Cluj-Napoca, Romania \\ ${ }^{c}$ Simion Stoilow Institute of Mathematics of the Romanian Academy, Research group of the project PD-3-0152, \\ P.O. Box 1-764, RO-014700 Bucharest, Romania \\ E-mail addresses: espinola@us.es (R. Espínola), anicolae@math.ubbcluj.ro (A. Nicolae)
}

\begin{abstract}
This paper deals with the study of parameter dependence of extensions of Lipschitz mappings from the point of view of continuity. We show that if assuming appropriate curvature bounds for the spaces, the multivalued extension operators that assign to every nonexpansive (resp. Lipschitz) mapping all its nonexpansive extensions (resp. Lipschitz extensions with the same Lipschitz constant) are lower semi-continuous and admit continuous selections. Moreover, we prove that Lipschitz mappings can be extended continuously even when imposing the condition that the image of the extension belongs to the closure of the convex hull of the image of the original mapping. When the target space is hyperconvex one can obtain in fact nonexpansivity.
\end{abstract}

Keywords: Lipschitz mapping, extension operator, continuous selection, geodesic space of bounded curvature, hyperconvexity

\section{Introduction}

The Kirszbraun theorem [12] is a fundamental result in the theory of Lipschitz extensions and states that for any Lipschitz function $f: A \subseteq \mathbb{R}^{n} \rightarrow \mathbb{R}^{m}$ there exists a Lipschitz extension $f^{\prime}: \mathbb{R}^{n} \rightarrow \mathbb{R}^{m}$ with the same Lipschitz constant. The result for arbitrary Hilbert spaces goes back to Valentine 22. Aronszajn and Panitchpakdi 3 introduced the concept of hyperconvexity, which is closely related to this problem since a metric space $Y$ is hyperconvex if and only if given any subspace $A$ of any metric space $X$, every nonexpansive mapping $f: A \rightarrow Y$ admits a nonexpansive extension to $X$.

The first result that extends Kirszbraun's theorem to the metric setting by imposing curvature bounds in the sense of Alexandrov was given by Lang and Schroeder in [17] (see also [16, 22] for previous particular results). The same problem was later approached by Alexander, Kapovitch and Petrunin in 2] where a different proof method is considered.

All the aforementioned results guarantee the existence of an extension for the original mapping. However, this extension is not necessary unique and no information is given on the parameter dependence of the extensions. Kopecká studied the process of assigning extensions to mappings from the point of view of continuity providing positive answers first in Euclidean [13] and then in Hilbert spaces [14. Namely, the multivalued extension mappings that assign to every nonexpansive (resp. Lipschitz) mapping all its nonexpansive extensions (resp. Lipschitz extensions with the same Lipschitz constant) are proved to be lower semi-continuous using Kirszbraun's theorem and a homotopy argument. Applying Michael's selection theorem one obtains continuous selections of these multivalued extension operators. Kopecká and Reich further generalized these results in [15, obtaining a continuous singlevalued extension operator with the additional condition that the image of the extension belongs to the closed convex hull of the image of the original mapping.

A natural question is to study this problem in geodesic metric spaces with curvature bounds in the sense of Alexandrov since in this context a generalized version of Kirszbraun's theorem holds. Here we show that one can indeed prove counterparts of such continuity results in this setting. In Section 3 we show that assuming appropriate curvature bounds for the spaces, the multivalued extension mappings are lower 
semi-continuous and admit continuous selections. Moreover, we prove in Section 4 that Lipschitz mappings can be extended continuously even when imposing the above mentioned convexity condition on the image of the extension. Section 5 deals with the case where the target space is hyperconvex and shows that in this situation one can obtain in fact nonexpansivity.

\section{Preliminaries}

Let $(X, d)$ be a metric space. A geodesic path from $x$ to $y$ is a mapping $c:[0, l] \subseteq \mathbb{R} \rightarrow X$ such that $c(0)=x, c(l)=y$ and $d\left(c(t), c\left(t^{\prime}\right)\right)=\left|t-t^{\prime}\right|$ for every $t, t^{\prime} \in[0, l]$. The image $c([0, l])$ of $c$ forms a geodesic segment which joins $x$ and $y$. Note that a geodesic segment from $x$ to $y$ is not necessarily unique. $(X, d)$ is a geodesic space if every two points in $X$ can be joined by a geodesic path. A point $z \in X$ belongs to a geodesic segment joining $x$ and $y$ if and only if there exists $t \in[0,1]$ such that $d(z, x)=t d(x, y)$ and $d(z, y)=(1-t) d(x, y)$, and we will write $z=(1-t) x+t y$ for simplicity. For more details on geodesic metric spaces the reader may check 4 .

A geodesic space $(X, d)$ is Busemann convex if given any pair of geodesic paths $c_{1}:\left[0, l_{1}\right] \rightarrow X$ and $c_{2}:\left[0, l_{2}\right] \rightarrow X$ with $c_{1}(0)=c_{2}(0)$ one has

$$
d\left(c_{1}\left(t l_{1}\right), c_{2}\left(t l_{2}\right)\right) \leq t d\left(c_{1}\left(l_{1}\right), c_{2}\left(l_{2}\right)\right), \quad \text { for every } t \in[0,1] .
$$

A subset $C$ of $X$ is convex if any geodesic segment that joins every two points of $C$ is contained in $C$. Let $G_{1}(C)$ denote the union of all geodesics segments with endpoints in $C$. Note that $C$ is convex if and only if $G_{1}(C)=C$. Recursively, for $n \geq 2$ we set $G_{n}(C)=G_{1}\left(G_{n-1}(C)\right)$. The convex hull of $C$ is

$$
\operatorname{co}(C)=\bigcup_{n \in \mathbb{N}} G_{n}(C) \text {. }
$$

By $\overline{\mathrm{co}}(C)$ we denote the closure of the convex hull. It is easy to see that in a Busemann convex geodesic space, the closure of the convex hull is convex and hence it is the smallest closed convex set containing $C$.

For $\kappa \in \mathbb{R}$ let $M_{\kappa}^{2}$ denote the complete, simply connected model surface of constant curvature $\kappa$. In the sequel we assume that $\kappa \leq 0$.

A geodesic triangle $\Delta=\Delta\left(x_{1}, x_{2}, x_{3}\right)$ consists of three points $x_{1}, x_{2}$ and $x_{3}$ in $X$ and three geodesic segments corresponding to each pair of points. A $\kappa$-comparison triangle for $\Delta$ is a triangle $\bar{\Delta}=\Delta\left(\bar{x}_{1}, \bar{x}_{2}, \bar{x}_{3}\right)$ in $M_{\kappa}^{2}$ such that $d\left(x_{i}, x_{j}\right)=d_{M_{\kappa}^{2}}\left(\bar{x}_{i}, \bar{x}_{j}\right)$ for $i, j \in\{1,2,3\}$. For $\kappa$ fixed, $\kappa$-comparison triangles of geodesic triangles always exist and are unique up to isometry.

A geodesic triangle $\Delta$ satisfies the $C A T(\kappa)$ (resp. reversed $C A T(\kappa)$ ) inequality if for every $\kappa$-comparison triangle $\bar{\Delta}$ of $\Delta$ and for every $x, y \in \Delta$ we have

$$
d(x, y) \leq d_{M_{\kappa}^{2}}(\bar{x}, \bar{y})\left(\operatorname{resp} . d(x, y) \geq d_{M_{\kappa}^{2}}(\bar{x}, \bar{y})\right)
$$

where $\bar{x}, \bar{y} \in \bar{\Delta}$ are the corresponding points of $x$ and $y$, i.e., if $x=(1-t) x_{i}+t x_{j}$ then $\bar{x}=(1-t) \bar{x}_{i}+t \bar{x}_{j}$.

A $C A T(\kappa)$ space (also known as a space of curvature bounded above by $\kappa$ in the sense of Alexandrov) is a geodesic space for which every geodesic triangle satisfies the CAT $(\kappa)$ inequality. Any CAT $(0)$ space (and so any $\mathrm{CAT}(\kappa)$ space) is Busemann convex.

A geodesic metric space is said to have curvature bounded below by $\kappa$ in the sense of Alexandrov (denoted by $C B B(\kappa))$ if every geodesic triangle satisfies the reversed $\operatorname{CAT}(\kappa)$ inequality. If $X$ is a $\operatorname{CBB}(\kappa)$ space, then the direct product $X \times M_{\kappa}^{2}$ is a $\mathrm{CBB}(\kappa)$ space with the metric

$$
d((x, a),(y, b))^{2}=d_{X}(x, y)^{2}+d_{M_{\kappa}^{2}}(a, b)^{2} .
$$

Other properties of spaces with curvature bounded above or below and equivalent definitions can be found in [4, [5].

Let $(X, d)$ be a metric space. Taking $x \in X$ and $r>0$ we denote the closed ball centered at $x$ with radius $r$ by $B(z, r)$. Given $C$ a nonempty subset of $X$, the distance of a point $x \in X$ to $C$ is $\operatorname{dist}(x, C)=$ $\inf \{d(x, c): c \in C\}$. If $B$ and $C$ are nonempty subsets of $X$, one defines the Pompeiu-Hausdorff distance as

$$
H(B, C)=\max \left\{\sup _{b \in B} \operatorname{dist}(b, C), \sup _{c \in C} \operatorname{dist}(c, B)\right\} .
$$


The metric projection $P_{C}$ onto $C$ is the mapping

$$
P_{C}(x)=\{c \in C: d(x, c)=\operatorname{dist}(x, C)\}, \quad \text { for every } x \in X .
$$

In any $\mathrm{CAT}(0)$ space the metric projection onto a convex and complete subset is a singlevalued and nonexpansive (that is, 1-Lipschitz) mapping.

A metric space $X$ is hyperconvex if $\bigcap_{\alpha} B\left(x_{\alpha}, r_{\alpha}\right) \neq \emptyset$ for every collection of points $\left\{x_{\alpha}\right\}$ in $X$ and positive numbers $\left\{r_{\alpha}\right\}$ such that $d\left(x_{\alpha}, x_{\beta}\right) \leq r_{\alpha}+r_{\beta}$ for any $\alpha, \beta$. A subset $E$ of a metric space $X$ is called externally hyperconvex (with respect to $X$ ) if given any family $\left\{x_{\alpha}\right\}$ of points in $X$ and any family $\left\{r_{\alpha}\right\}$ of real numbers satisfying

$$
d\left(x_{\alpha}, x_{\beta}\right) \leq r_{\alpha}+r_{\beta} \quad \text { and } \quad \operatorname{dist}\left(x_{\alpha}, E\right) \leq r_{\alpha}
$$

it follows that $\bigcap_{\alpha} B\left(x_{\alpha}, r_{\alpha}\right) \cap E \neq \emptyset$. For a more detailed discussion on hyperconvex metric spaces, see [6].

Let $\left(X, d_{X}\right),\left(Y, d_{Y}\right)$ be metric spaces, $A \subseteq X$ nonempty and consider $C(A, Y)$ the family of bounded and continuous mappings from $A$ to $Y$. For each $f, g \in C(A, Y)$, let $d_{\infty}(f, g)=\sup _{x \in A} d_{Y}(f(x), g(x))$. Endowed with the supremum distance $d_{\infty}, C(A, Y)$ is a metric space which is complete if $Y$ is complete. We consider two subsets of $C(A, Y): \mathscr{L}(A, Y)$ which includes all bounded Lipschitz mappings from $A$ to $Y$ and is not necessarily a closed subset of $C(A, Y)$ and $\mathscr{N}(A, Y)$ which stands for the family of all bounded nonexpansive mappings defined from $A$ to $Y$ and which is closed in $C(A, Y)$.

For $f \in \mathscr{L}(A, Y)$ we denote the smallest Lipschitz constant of $f$ on $B \subseteq A$ by $\operatorname{Lip}(f, B)$. More precisely,

$$
\operatorname{Lip}(f, B)=\sup \left\{\frac{d_{Y}(f(x), f(y))}{d_{X}(x, y)}: x, y \in B, x \neq y\right\} .
$$

For a set $C$, we denote by $\mathcal{P}(C)$ the family of all its subsets. We consider two multivalued extension mappings:

- $\Phi: \mathscr{N}(A, Y) \rightarrow \mathcal{P}(\mathscr{N}(X, Y))$ which assigns to each nonexpansive mapping $f \in \mathscr{N}(A, Y)$ all its nonexpansive extensions $f^{\prime} \in \mathscr{N}(X, Y)$. Note that in this case it may happen that $\operatorname{Lip}(f, A)<$ $\operatorname{Lip}\left(f^{\prime}, X\right) \leq 1$.

- $\Psi: \mathscr{L}(A, Y) \rightarrow \mathcal{P}(\mathscr{L}(X, Y))$ which assigns to each Lipschitz mapping $f \in \mathscr{L}(A, Y)$ all its Lipschitz extensions $f^{\prime} \in \mathscr{L}(X, Y)$ with $\operatorname{Lip}(f, A)=\operatorname{Lip}\left(f^{\prime}, X\right)$.

Recall that having two topological spaces $X$ and $Y$, a multivalued mapping $\Gamma: X \rightarrow \mathcal{P}(Y)$ is lower semi-continuous if for every open $V \subseteq Y$, the set $\{x \in X: \Gamma(x) \cap V \neq \emptyset\}$ is open in $X$. If $X$ and $Y$ are metric spaces, $\Gamma$ is nonexpansive if $H(\Gamma(x), \Gamma(y)) \leq d_{X}(x, y)$ for every $x, y \in X$.

The classical Kirszbraun theorem was extended to geodesic metric spaces with lower and upper curvature bounds by Lang and Schroeder in [17. Later, Alexander, Kapovitch and Petrunin considered a different approach of the proof in [2].

Theorem 2.1 (Lang, Schroeder [17]). Let $\kappa \leq 0, X$ a $C B B(\kappa)$ space and $Y$ a complete CAT $(\kappa)$ space. Suppose $A \subseteq X$ is nonempty and $f: A \rightarrow Y$ is nonexpansive. Then there exists a nonexpansive extension $f^{\prime}: X \rightarrow Y$ of $f$

Although the result can be also stated when $\kappa>0$ with an appropriate boundedness condition on the set $f(A)$, here we are only concerned with the case $\kappa \leq 0$.

For $\kappa=0$ the result can be generalized to any arbitrary Lipschitz constant by scaling the metric on either $X$ or $Y$ and so we may consider both mappings $\Phi$ and $\Psi$. When $\kappa<0$, the same argument can be applied for Lipschitz constants greater than 1. However, for Lipschitz constants strictly less than 1 , we cannot expect the result to hold true. Suppose one could extend all mappings $f: A \subseteq \mathbb{H}^{2} \rightarrow \mathbb{H}^{2}$ with $\operatorname{Lip}(f, A)<1$ while keeping the same Lipschitz constant. Taking $\kappa \in(-1,0)$, this implies that we can extend all nonexpansive mappings defined on $A \subseteq \mathbb{H}^{2}$ with values in $M_{\kappa}^{2}$ to nonexpansive mappings on $\mathbb{H}^{2}$. But this means that $M_{\kappa}^{2}$ is a CAT $(-1)$ space (see Proposition 6.2 in [17]), a contradiction. Since in this work we rely on Theorem 2.1 in order to obtain our continuity results, for the case $\kappa<0$ we will only study the mapping $\Phi$.

However, if the target space is an $\mathbb{R}$-tree, then it was proved in [17] that we not only can extend mappings with arbitrary Lipschitz constant, but we can also drop the curvature assumption on the source space. 
Theorem 2.2 (Lang, Schroeder [17]). Let $X$ be a metric space and $Y$ a complete $\mathbb{R}$-tree. Suppose $A \subseteq X$ is nonempty and $f: A \rightarrow Y$ is a Lipschitz mapping. Then there exists a Lipschitz extension $f^{\prime}: X \rightarrow Y$ of $f$ with $\operatorname{Lip}\left(f^{\prime}, X\right)=\operatorname{Lip}(f, A)$.

Theorem 2.2 is a consequence of the following extension theorem proved for hyperconvex metric spaces by Aronszajn and Panitchpakdi in [3], where it is actually shown that this property characterizes hyperconvexity. Note that any complete $\mathbb{R}$-tree is a hyperconvex metric space (see [11]).

Theorem 2.3 (Aronszajn, Panitchpakdi [3]). Let $X$ be a metric space and $Y$ a hyperconvex metric space. Suppose $A \subseteq X$ is nonempty and $f: A \rightarrow Y$ is a Lipschitz mapping. Then there exists a Lipschitz extension $f^{\prime}: X \rightarrow Y$ of $f$ with $\operatorname{Lip}\left(f^{\prime}, X\right)=\operatorname{Lip}(f, A)$.

\section{Lower semicontinuity of the multivalued extension mappings and continuous selections}

We begin this section by showing that, when considering appropriate curvature bounds on $X$ and $Y$, both mappings $\Phi$ and $\Psi$ are lower semi-continuous which is an immediate consequence of Lemmas 3.1 and 3.2 . respectively. The proof strategy follows the one used for Hilbert spaces in [14].

Lemma 3.1. Let $\kappa \leq 0, X$ a $C B B(\kappa)$ space, $Y$ a complete $C A T(\kappa)$ space and $A \subseteq X$ nonempty. Let $f \in$ $\mathscr{N}(X, Y)$. Then for every $\varepsilon>0$ there exists $\delta>0$ such that every $g \in \mathscr{N}(A, Y)$ with $\sup _{a \in A} d_{Y}(f(a), g(a))<$ $\delta$ admits an extension $g^{\prime} \in \mathscr{N}(X, Y)$ such that $d_{\infty}\left(f, g^{\prime}\right) \leq \varepsilon$.

Proof. Since $f$ is a bounded mapping there exists $z \in Y$ and $M \geq 1$ such that $\sup _{x \in X} d_{Y}(z, f(x)) \leq M$. Let $\varepsilon \in(0,1)$ and take $\delta=\varepsilon^{2} /(8 M)$. Suppose $g \in \mathscr{N}(A, Y)$ with $\sup _{a \in A} d_{Y}(f(a), g(a))<\delta$.

Let $\kappa=0$. Define the mapping $h: X \times\{(0,0)\} \cup A \times\{(0, \varepsilon)\} \rightarrow Y$ by: for $x \in X, h(x,(0,0))=f(x)$ and for $a \in A, h(a,(0, \varepsilon))=g(a)$.

Recalling (11), for $x \in X$ and $a \in A$,

$$
\begin{aligned}
d_{Y} & (h(x,(0,0)), h(a,(0, \varepsilon)))^{2}=d_{Y}(f(x), g(a))^{2} \\
& \leq\left(d_{Y}(f(x), f(a))+d_{Y}(f(a), g(a))\right)^{2} \\
& \leq d_{X}(x, a)^{2}+\delta^{2}+4 \delta M<d_{X}(x, a)^{2}+\varepsilon^{2}=d((x,(0,0)),(a,(0, \varepsilon)))^{2} .
\end{aligned}
$$

This shows that $h$ is nonexpansive since both $f$ and $g$ are nonexpansive on $X$ and $A$, respectively. Since $X \times \mathbb{R}^{2}$ is a $\mathrm{CBB}(0)$ space, using Theorem 2.1 we can extend $h$ to a nonexpansive mapping $h^{\prime}: X \times \mathbb{R}^{2} \rightarrow Y$. Define $g^{\prime}: X \rightarrow Y$ by $g^{\prime}(x)=h^{\prime}(x,(0, \varepsilon))$. Clearly, $g^{\prime}$ is nonexpansive and coincides with $g$ on $A$. Moreover, for each $x \in X$,

$$
d_{Y}\left(f(x), g^{\prime}(x)\right)=d_{Y}\left(h^{\prime}(x,(0,0)), h^{\prime}(x,(0, \varepsilon))\right) \leq d((x,(0,0)),(x,(0, \varepsilon)))=\varepsilon .
$$

This also shows that $g^{\prime}$ is bounded.

When $\kappa<0$, we apply the same argument to the nonexpansive mapping

$$
h: X \times\{(0,0,1)\} \cup A \times\{(0, \sinh (\sqrt{-\kappa} \varepsilon), \cosh (\sqrt{-\kappa} \varepsilon))\} \rightarrow Y
$$

defined as: for $x \in X, h(x,(0,0,1))=f(x)$ and for $a \in A, h(a,(0, \sinh (\sqrt{-\kappa} \varepsilon), \cosh (\sqrt{-\kappa} \varepsilon)))=g(a)$ which can be extended to a nonexpansive mapping $h^{\prime}: X \times M_{\kappa}^{2} \rightarrow Y$ (recall that $X \times M_{\kappa}^{2}$ is a $\mathrm{CBB}(\kappa)$ space).

Lemma 3.2. Let $X$ be a $C B B(0)$ space, $Y$ a complete $C A T(0)$ space and $A \subseteq X$ nonempty. Let $f \in \mathscr{L}(X, Y)$ with $\operatorname{Lip}(f, A)=\operatorname{Lip}(f, X)$. Then for every $\varepsilon>0$ there exists $\delta>0$ such that every $g \in \mathscr{L}(A, Y)$ for which $\sup _{a \in A} d_{Y}(f(a), g(a))<\delta$ admits an extension $g^{\prime} \in \mathscr{L}(X, Y)$ with $\operatorname{Lip}(g, A)=\operatorname{Lip}\left(g^{\prime}, X\right)$ and $d_{\infty}\left(f, g^{\prime}\right) \leq \varepsilon$. 
Proof. Let $\varepsilon \in(0,1)$. Suppose first that $f$ is constant and equal to some $y \in Y$. Let $\delta=\varepsilon$. Then having any extension $g_{1}$ of $g$ to $X$ with $\operatorname{Lip}(g, A)=\operatorname{Lip}\left(g_{1}, X\right)$, we can take $g^{\prime}: X \rightarrow Y, g^{\prime}(x)=P_{B(y, \varepsilon)} \circ g_{1}$.

Assume now $f$ is not constant. Let $z \in Y$ and $M>0$ such that

$$
\sup _{x \in X} d_{Y}(z, f(x)) \leq M
$$

Let $s \in(0,1)$ for which

$$
\frac{1-s}{s^{2}}<\frac{\varepsilon^{2}}{32 M(4 M+1)}
$$

Since $\operatorname{Lip}(f, A)=\operatorname{Lip}(f, X)>0$, there exist $x_{0}, y_{0} \in A$ such that $d_{Y}\left(f\left(x_{0}\right), f\left(y_{0}\right)\right)>s \operatorname{Lip}(f, X) d_{X}\left(x_{0}, y_{0}\right)$. Take

$$
\delta=\min \left\{\frac{d_{Y}\left(f\left(x_{0}\right), f\left(y_{0}\right)\right)-s \operatorname{Lip}(f, X) d_{X}\left(x_{0}, y_{0}\right)}{2}, \frac{\varepsilon^{2} s^{2}}{32(4 M+1)}\right\} .
$$

Let $g \in \mathscr{L}(A, Y)$ with $\sup _{a \in A} d_{Y}(f(a), g(a))<\delta$.

Suppose first $\operatorname{Lip}(g, A) \leq 2 \operatorname{Lip}(f, X)$. Then,

$$
\begin{aligned}
d_{Y}\left(g\left(x_{0}\right), g\left(y_{0}\right)\right) & \geq d_{Y}\left(f\left(x_{0}\right), f\left(y_{0}\right)\right)-d_{Y}\left(f\left(x_{0}\right), g\left(x_{0}\right)\right)-d_{Y}\left(f\left(y_{0}\right), g\left(y_{0}\right)\right) \\
& >d_{Y}\left(f\left(x_{0}\right), f\left(y_{0}\right)\right)-2 \delta \geq s \operatorname{Lip}(f, X) d_{X}\left(x_{0}, y_{0}\right),
\end{aligned}
$$

from where $\operatorname{Lip}(g, A) \geq s \operatorname{Lip}(f, X)$. Let $\eta=\varepsilon /(4 \operatorname{Lip}(f, X))$ and $h: X \times\{(0,0)\} \cup A \times\{(0, \eta)\} \rightarrow Y$ be defined by: for $x \in X, h(x,(0,0))=(1-s) z+s f(x)$ and for $a \in A, h(a,(0, \eta))=g(a)$. Thus, for $x \in X$ and $a \in A$ we have that

$$
\begin{aligned}
d_{Y} & (h(x,(0,0)), h(a,(0, \eta)))^{2}=d_{Y}((1-s) z+s f(x), g(a))^{2} \\
\leq & \left(d_{Y}((1-s) z+s f(x), f(a))+d_{Y}(f(a), g(a))\right)^{2} \\
\leq & \left((1-s) M+s d_{Y}(f(x), f(a))+\delta\right)^{2} \\
\leq & (\delta+(1-s) M)^{2}+s^{2} \operatorname{Lip}(f, X)^{2} d_{X}(x, a)^{2}+4 s M(\delta+(1-s) M) \\
< & s^{2} \operatorname{Lip}(f, X)^{2}\left(d_{X}(x, a)^{2}+\frac{(\delta+(1-s) M)(4 M+1)}{s^{2} \operatorname{Lip}(f, X)^{2}}\right) \\
& \quad \operatorname{since}(\delta+(1-s) M)^{2}<\delta+(1-s) M \text { and } s<1 \\
< & s^{2} \operatorname{Lip}(f, X)^{2}\left(d_{X}(x, a)^{2}+\eta^{2}\right) \\
& \quad \operatorname{since} \delta+(1-s) M<\varepsilon^{2} s^{2} /(16(4 M+1)) \\
\leq & \operatorname{Lip}(g, A)^{2} d((x,(0,0)),(a,(0, \eta)))^{2} .
\end{aligned}
$$

To complete the argument that $h$ is Lipschitz with smallest Lipschitz constant $\operatorname{Lip}(g, A)$ one uses Busemann convexity in $Y$ along with the fact that the mappings $f$ and $g$ are $\operatorname{Lipschitz}$ and $\operatorname{Lip}(g, A) \geq s \operatorname{Lip}(f, X)$. Since $X \times \mathbb{R}^{2}$ is a $\operatorname{CBB}(0)$ space, by Theorem 2.1 we can extend $h$ to a Lipschitz mapping $h^{\prime}: X \times \mathbb{R}^{2} \rightarrow Y$ with $\operatorname{Lip}\left(h^{\prime}, X \times \mathbb{R}^{2}\right)=\operatorname{Lip}(g, A)$. Define $g^{\prime}: X \rightarrow Y$ by $g^{\prime}(x)=h^{\prime}(x,(0, \eta))$. Clearly, $g^{\prime}$ extends $g$ and $\operatorname{Lip}\left(g^{\prime}, X\right)=\operatorname{Lip}(g, A)$. Moreover, for every $x \in X$,

$$
\begin{aligned}
d_{Y}\left(g^{\prime}(x), f(x)\right) & \leq d_{Y}\left(g^{\prime}(x),(1-s) z+s f(x)\right)+d_{Y}((1-s) z+s f(x), f(x)) \\
& \leq d_{Y}\left(h^{\prime}(x,(0, \eta)), h^{\prime}(x,(0,0))\right)+(1-s) M<\operatorname{Lip}(g, A) \eta+\varepsilon / 2 \\
& \leq 2 \operatorname{Lip}(f, X) \frac{\varepsilon}{4 \operatorname{Lip}(f, X)}+\frac{\varepsilon}{2}=\varepsilon .
\end{aligned}
$$

If $\operatorname{Lip}(g, A)>2 \operatorname{Lip}(f, X)$, consider the set

$$
\tilde{A}=\left\{x \in X: \operatorname{dist}(x, A) \geq \frac{2 \delta}{\operatorname{Lip}(g, A)}\right\}
$$


and define the mapping $\tilde{g}: A \cup \tilde{A} \rightarrow Y$ by: for $a \in A, \tilde{g}(a)=g(a)$ and for $x \in \tilde{A}, \tilde{g}(x)=f(x)$. To see that $\operatorname{Lip}(g, A)=\operatorname{Lip}(\tilde{g}, A \cup \tilde{A})$ it suffices to verify that for any $a \in A$ and $x \in \tilde{A}$,

$$
\begin{aligned}
d_{Y}(\tilde{g}(x), \tilde{g}(a)) & =d_{Y}(f(x), g(a)) \leq d_{Y}(f(x), f(a))+d_{Y}(f(a), g(a)) \\
& <\frac{\operatorname{Lip}(g, A)}{2} d_{X}(x, a)+\delta \leq \frac{\operatorname{Lip}(g, A)}{2} d_{X}(x, a)+\frac{\operatorname{Lip}(g, A)}{2} \operatorname{dist}(x, A) \\
& \leq \operatorname{Lip}(g, A) d_{X}(x, a) .
\end{aligned}
$$

Take $g^{\prime}$ to be any extension of $\tilde{g}$ for which $\operatorname{Lip}(g, A)=\operatorname{Lip}\left(g^{\prime}, X\right)$. For $x \in \tilde{A}, f(x)=g^{\prime}(x)$. If $x \notin \tilde{A}$, there exists $a \in A$ such that $d_{X}(x, a)<2 \delta / \operatorname{Lip}(g, A)$. Thus,

$$
\begin{aligned}
d_{Y}\left(f(x), g^{\prime}(x)\right) & \leq d_{Y}(f(x), f(a))+d_{Y}\left(f(a), g^{\prime}(a)\right)+d_{Y}\left(g^{\prime}(a), g^{\prime}(x)\right) \\
& <\frac{\operatorname{Lip}(g, A)}{2} \frac{2 \delta}{\operatorname{Lip}(g, A)}+\delta+\operatorname{Lip}(g, A) \frac{2 \delta}{\operatorname{Lip}(g, A)}=4 \delta<\varepsilon .
\end{aligned}
$$

This ends the proof.

Theorem 3.3. Let $\kappa \leq 0, X$ a $C B B(\kappa)$ space, $Y$ a complete $C A T(\kappa)$ space and $A \subseteq X$ nonempty. Then the mapping $\Phi: \mathscr{N}(A, Y) \rightarrow \mathcal{P}(\mathscr{N}(X, Y))$ is lower semi-continuous.

Theorem 3.4. Let $X$ be a $C B B(0)$ space, $Y$ a complete $C A T(0)$ space and $A \subseteq X$ nonempty. Then the mapping $\Psi: \mathscr{L}(A, Y) \rightarrow \mathcal{P}(\mathscr{L}(X, Y))$ is lower semi-continuous.

Using the lower semi-continuity of the mappings $\Phi$ and $\Psi$ we prove that they admit continuous selections. In order to obtain these singlevalued continuous extension operators we apply a selection result due to Horvath 8] which is a generalization of the classical Michael selection theorem to the setting of $c$-spaces. Before stating this selection result we recall the following notions: for $Z$ a topological space, denote by $\langle Z\rangle$ the family of its nonempty and finite subsets. A mapping $F:\langle Z\rangle \rightarrow \mathcal{P}(Z)$ is a c-structure if firstly, for each $A \in\langle Z\rangle, F(A)$ is nonempty and contractible, and secondly, for every $A_{1}, A_{2} \in\langle Z\rangle, A_{1} \subseteq A_{2}$ implies $F\left(A_{1}\right) \subseteq F\left(A_{2}\right)$. The pair $(Z, F)$ is called a $c$-space and $V \subseteq Z$ is an $F$-set if for every $A \in\langle V\rangle$ we have that $F(A) \subseteq V$. A $c$-space $(Z, F)$ is called an l.c. metric space is $(Z, d)$ is a metric space such that open balls are $F$-sets and if $V \subseteq Z$ is an $F$-set, then for every $\varepsilon>0,\{z \in Z: \operatorname{dist}(z, V)<\varepsilon\}$ is an $F$-set. The selection result that we apply is the following.

Theorem 3.5 (Horvath [8]). Let $U$ be a paracompact topological space, $(Z, F)$ an l.c. complete metric space and $\Gamma: U \rightarrow \mathcal{P}(Z)$ lower semi-continuous such that for each $u \in U, \Gamma(u)$ is a nonempty and closed $F$-set. Then there exists a continuous selection for $\Gamma$.

Let $\kappa \leq 0$. Suppose $X$ is a $\operatorname{CBB}(\kappa)$ space and $Y$ a complete $\operatorname{CAT}(\kappa)$ space. We check in the sequel that we can indeed make use of the above theorem relying basically on Busemann convexity in $Y$. We say that $B \in \mathcal{P}(C(X, Y))$ is convex if for every $g_{1}, g_{2} \in B$ and every $t \in[0,1]$ we have that the mapping $h: X \rightarrow Y$, $h=(1-t) g_{1}+t g_{2}$ (that is, $h(x)=(1-t) g_{1}(x)+t g_{2}(x)$ for every $\left.x \in X\right)$ belongs to $B$. Note that balls in $C(X, Y)$ are convex.

The mapping $\Phi$ has nonempty and closed values in $C(X, Y)$. Moreover, for each $f \in \mathscr{N}(A, Y), \Phi(f)$ is convex. To see this let $f^{\prime}, f^{\prime \prime} \in \Phi(f)$ and $t \in[0,1]$. Then, for each $x \in X$,

$$
\begin{aligned}
d_{Y}\left((1-t) f^{\prime}(x)+t f^{\prime \prime}(x),(1-t) f^{\prime}(y)+t f^{\prime \prime}(y)\right) \leq & (1-t) d_{Y}\left(f^{\prime}(x), f^{\prime}(y)\right) \\
& +t d_{Y}\left(f^{\prime \prime}(x), f^{\prime \prime}(y)\right) \\
\leq & d_{X}(x, y) .
\end{aligned}
$$

Similarly, when $\kappa=0, \Psi$ is also nonempty, closed and convex-valued. Define $F:\langle C(X, Y)\rangle \rightarrow \mathcal{P}(C(X, Y))$ by

$$
F(A)=\bigcap\{B: A \subseteq B, B \text { convex }\}, \quad \text { for each } A \in\langle C(X, Y)\rangle .
$$

Let $A \in\langle C(X, Y)\rangle$. Then $F(A) \neq \emptyset$. Fix $g_{1} \in A$ and define $H:[0,1] \times F(A) \rightarrow F(A)$ by $H(t, f)=$ $(1-t) f+t g_{1}$. Note that for each $f \in F(A), H(0, f)=f$ and $H(1, f)=g_{1}$. It is easy to see that 
$H$ is continuous and so $F(A)$ is contractible. Clearly, for every $A_{1}, A_{2} \in\langle C(X, Y)\rangle, A_{1} \subseteq A_{2}$ implies $F\left(A_{1}\right) \subseteq F\left(A_{2}\right)$. Thus, $(C(X, Y), F)$ is a $c$-space. Note that a subset of $C(X, Y)$ is an $F$-set if and only if it is convex. By Busemann convexity in $Y$ one can finally show that $(C(X, Y), F)$ is an l.c. metric space.

Theorem 3.6. Let $\kappa \leq 0, X$ a $C B B(\kappa)$ space, $Y$ a complete $C A T(\kappa)$ space and $A \subseteq X$ nonempty. Then there exists a continuous mapping $\alpha: \mathscr{N}(A, Y) \rightarrow \mathscr{N}(X, Y)$ such that for all $g \in \mathscr{N}(A, Y), \alpha(g)(a)=g(a)$ for every $a \in A$.

Proof. We can view the mapping $\Phi$ with values in $\mathcal{P}(C(X, Y))$ while still preserving its lower semi-continuity. Since any metric space is a paracompact topological space we can now apply Theorem 3.5 to obtain a continuous extension mapping $\alpha: \mathscr{N}(A, Y) \rightarrow C(X, Y)$. Because $\Phi$ actually takes values in $\mathcal{P}(\mathscr{N}(X, Y))$ we obtain the conclusion.

For bounded Lipschitz mappings we obtain the following result.

Theorem 3.7. Let $X$ be a $C B B(0)$ space, $Y$ a complete $C A T(0)$ space and $A \subseteq X$ nonempty. Then there exists a continuous mapping $\beta: \mathscr{L}(A, Y) \rightarrow \mathscr{L}(X, Y)$ such that for all $g \in \overline{\mathscr{L}}(A, Y), \beta(g)(a)=g(a)$ for every $a \in A$ and $\operatorname{Lip}(\beta(g), X)=\operatorname{Lip}(g, A)$.

Remark 3.8. Note that in Lemmas 3.1 and 3.2 the lower curvature bound of $X$ is only used to apply Theorem [2.1, However, when $Y$ is a complete $\mathbb{R}$-tree, one can extend Lipschitz mappings (while keeping the same Lipschitz constant) if $X$ is an arbitrary metric space. Thus, as before, one can consider even a simpler reasoning in $X \times \mathbb{R}$ to obtain that both mappings $\Phi$ and $\Psi$ are lower semi-continuous and admit continuous selections. This property will be improved for the mapping $\Phi$ in Section 5 .

Remark 3.9. If $\kappa>0$ the argument given in this section does not work in a straightforward way. Note that in this case the direct product $X \times M_{\kappa}^{2}$ is not necessarily a $C B B(\kappa)$ space. Moreover, the images of the mappings $\Phi$ and $\Psi$ are no longer $F$-sets when considering the c-structure $F$ defined before.

\section{A convexity assumption on the images of the extensions}

In this section we show that one can actually choose extensions in a continuous way even when imposing the condition that the image of the extension belongs to the closure of the convex hull of the image of the original mapping. Related results in the case of Hilbert spaces were recently established in [15], and we extend them to our setting.

Recall first the next inequality which stems from the work of Reshetnyak (see, for instance, [9, Theorem 2.3.1] or [18, Lemma 2.1] for a simple proof).

Lemma 4.1. Let $Y$ be a $C A T(0)$ space. Then for every $x, y, u, v \in Y$,

$$
d(x, y)^{2}+d(u, v)^{2} \leq d(x, v)^{2}+d(y, u)^{2}+2 d(x, u) d(y, v) .
$$

The property below provides a uniform bound on the distance between the projection points from a common point onto two sets. A similar result in uniformly smooth Banach spaces is [1, Lemma 3.4].

Lemma 4.2. Let $Y$ be a complete $C A T(0)$ space, $C_{1}, C_{2} \subseteq Y$ nonempty, closed and convex and suppose $r_{1}$ and $r_{2}$ are positive numbers. If there exists $z \in Y$ such that $C_{1}, C_{2} \subseteq B\left(z, r_{1}\right)$, then for any $x \in B\left(z, r_{2}\right)$,

$$
d\left(P_{C_{1}}(x), P_{C_{2}}(x)\right)^{2} \leq 2\left(r_{1}+r_{2}\right) H\left(C_{1}, C_{2}\right) .
$$

Proof. Let $C_{1}, C_{2} \subseteq B\left(z, r_{1}\right)$ and $x \in B\left(z, r_{2}\right)$. Denote $p_{1}=P_{C_{1}}(x), p_{2}=P_{C_{2}}(x), q_{1}=P_{C_{1}}\left(p_{2}\right)$ and $q_{2}=P_{C_{2}}\left(p_{1}\right)$. Clearly, $d\left(p_{2}, q_{1}\right) \leq H\left(C_{1}, C_{2}\right)$ and $d\left(p_{1}, q_{2}\right) \leq H\left(C_{1}, C_{2}\right)$. Note also that that $d\left(x, p_{1}\right) \leq$ $d(x, z)+d\left(z, p_{1}\right) \leq r_{1}+r_{2}$ and $d\left(x, p_{2}\right) \leq r_{1}+r_{2}$. Since $q_{1} \in C_{1}$ and $p_{1}=P_{C_{1}}(x)$ it follows that $\angle_{p_{1}}\left(x, q_{1}\right) \geq$ $\pi / 2$ (see 4, Proposition 2.4, page 176]) which yields $d\left(x, q_{1}\right)^{2} \geq d\left(x, p_{1}\right)^{2}+d\left(p_{1}, q_{1}\right)^{2}$. Similarily, $d\left(x, q_{2}\right)^{2} \geq$ $d\left(x, p_{2}\right)^{2}+d\left(p_{2}, q_{2}\right)^{2}$. By Lemma 4.1 we also have that

$$
d\left(x, q_{1}\right)^{2}+d\left(p_{1}, p_{2}\right)^{2} \leq d\left(x, p_{2}\right)^{2}+d\left(p_{1}, q_{1}\right)^{2}+2 d\left(x, p_{1}\right) d\left(p_{2}, q_{1}\right),
$$


and therefore

$$
d\left(x, p_{1}\right)^{2}+d\left(p_{1}, p_{2}\right)^{2} \leq d\left(x, p_{2}\right)^{2}+2 d\left(x, p_{1}\right) d\left(p_{2}, q_{1}\right) .
$$

Likewise,

$$
d\left(x, p_{2}\right)^{2}+d\left(p_{1}, p_{2}\right)^{2} \leq d\left(x, p_{1}\right)^{2}+2 d\left(x, p_{2}\right) d\left(p_{1}, q_{2}\right) .
$$

Adding (2) and (3) we get that

$$
d\left(p_{1}, p_{2}\right)^{2} \leq d\left(x, p_{1}\right) d\left(p_{2}, q_{1}\right)+d\left(x, p_{2}\right) d\left(p_{1}, q_{2}\right) \leq 2\left(r_{1}+r_{2}\right) H\left(C_{1}, C_{2}\right) .
$$

We prove next a property of the Hausdorff distance. For the corresponding result in the setting of normed spaces, see [19].

Lemma 4.3. Let $Y$ be a $C A T(0)$ space, $C_{1}, C_{2} \subseteq Y$ nonempty. Then,

$$
H\left(\overline{\mathrm{co}}\left(C_{1}\right), \overline{\mathrm{co}}\left(C_{2}\right)\right) \leq H\left(C_{1}, C_{2}\right) .
$$

Proof. Let $c \in C_{2}$. Obviously, dist $\left(c, \overline{\mathrm{co}}\left(C_{1}\right)\right) \leq \operatorname{dist}\left(c, C_{1}\right) \leq H\left(C_{1}, C_{2}\right)$. Consider the set

$$
E=\left\{y \in Y: \operatorname{dist}\left(y, \overline{\mathrm{co}}\left(C_{1}\right)\right) \leq H\left(C_{1}, C_{2}\right)\right\},
$$

which is a closed and convex set (by Busemann convexity). Since $C_{2} \subseteq E$ it follows that $\overline{\mathrm{co}}\left(C_{2}\right) \subseteq E$, from where $\sup _{c \in \overline{\mathrm{co}}\left(C_{2}\right)} \operatorname{dist}\left(c, \overline{\mathrm{co}}\left(C_{1}\right)\right) \leq H\left(C_{1}, C_{2}\right)$. In a similar way we have that $\sup _{c \in \overline{\mathrm{co}}\left(C_{1}\right)} \operatorname{dist}\left(c, \overline{\mathrm{co}}\left(C_{2}\right)\right) \leq$ $H\left(C_{1}, C_{2}\right)$ and we are done.

Theorem 4.4. Let $\kappa \leq 0, X$ a $C B B(\kappa)$ space, $Y$ a complete $C A T(\kappa)$ space and $A \subseteq X$ nonempty. Then there exists a continuous mapping $\alpha_{c}: \mathscr{N}(A, Y) \rightarrow \mathscr{N}(X, Y)$ such that for all $g \in \mathscr{N}(A, Y), \alpha_{c}(g)(a)=g(a)$ for every $a \in A$ and $\alpha_{c}(g)(X) \subseteq \overline{\mathrm{CO}}(g(A))$.

Proof. By Theorem 3.6 there exists a continuous $\alpha: \mathscr{N}(A, Y) \rightarrow \mathscr{N}(X, Y)$ such that for all $g \in \mathscr{N}(A, Y)$, $\alpha(g)$ extends $g$. Define a mapping $\alpha_{c}$ on $\mathscr{N}(A, Y)$ by

$$
\alpha_{c}(g)(x)=P_{\overline{\mathrm{co}}(g(A))}(\alpha(g)(x)), \quad \text { for each } g \in \mathscr{N}(A, Y) \text { and } x \in X .
$$

For each $g \in \mathscr{N}(A, Y), \alpha_{c}(g) \in \mathscr{N}(X, Y)$ since the projection onto complete and convex subsets is nonexpansive. Clearly, $\alpha_{c}(g)(X) \subseteq \overline{\mathrm{co}}(g(A))$ and $\alpha_{c}(g)$ coincides with $g$ on $A$.

Thus, we only need to prove that $\alpha_{c}$ is continuous. Let $f \in \mathscr{N}(A, Y)$ and $\varepsilon>0$. Since $\alpha$ is continuous, there exists $\delta_{1}<1$ such that for every $g \in \mathscr{N}(A, Y)$ with $d_{\infty}(f, g)<\delta_{1}$ we have that $d_{\infty}(\alpha(f), \alpha(g))<\varepsilon / 2$. Fix $z \in Y$. Let $r=\sup _{x \in X} d_{Y}(z, \alpha(f)(x))$ and take $\delta=\min \left\{\delta_{1}, \frac{\varepsilon^{2}}{16(r+1)}\right\}$. Let $g \in \mathscr{N}(A, Y)$ with $d_{\infty}(f, g)<\delta$. Then, for every $x \in X$,

$$
\begin{aligned}
d_{Y}\left(\alpha_{c}(f)(x), \alpha_{c}(g)(x)\right)= & d_{Y}\left(P_{\overline{\mathrm{co}}(f(A))}(\alpha(f)(x)), P_{\overline{\mathrm{co}}(g(A))}(\alpha(g)(x))\right) \\
\leq & d_{Y}\left(P_{\overline{\mathrm{co}}(f(A))}(\alpha(f)(x)), P_{\overline{\mathrm{Co}}(g(A))}(\alpha(f)(x))\right) \\
& +d_{Y}\left(P_{\overline{\mathrm{Co}}(g(A))}(\alpha(f)(x)), P_{\overline{\mathrm{co}}(g(A))}(\alpha(g)(x))\right) .
\end{aligned}
$$

Note that $\sup _{a \in A} d_{Y}(z, f(a)) \leq r$ and $\sup _{a \in A} d_{Y}(z, g(a)) \leq r+1$. Apply Lemma 4.2 with $C_{1}=\overline{\mathrm{co}}(f(A))$, $C_{2}=\overline{\mathrm{co}}(g(A))$ and $r_{1}=r_{2}=r+1$ to get that

$$
\begin{aligned}
d_{Y}\left(P_{\overline{\mathrm{co}}(f(A))}(\alpha(f)(x)), P_{\overline{\mathrm{co}}(g(A))}(\alpha(f)(x))\right) \leq & 2 \sqrt{r+1} \sqrt{H(\overline{\mathrm{co}}(f(A)), \overline{\mathrm{co}}(g(A)))} \\
\leq & 2 \sqrt{r+1} \sqrt{H(f(A), g(A))} \\
& \text { by Lemma } 4.3 \\
\leq & 2 \sqrt{r+1} \sqrt{\sup _{a \in A} d_{Y}(f(a), g(a))} \leq \varepsilon / 2 .
\end{aligned}
$$


At the same time,

$$
\begin{aligned}
d_{Y}\left(P_{\overline{\mathrm{Co}}(g(A))}(\alpha(f)(x)), P_{\overline{\mathrm{Co}}(g(A))}(\alpha(g)(x))\right) & \leq d_{Y}(\alpha(f)(x), \alpha(g)(x)) \\
& \leq d_{\infty}(\alpha(f), \alpha(g))<\varepsilon / 2 .
\end{aligned}
$$

Hence, $d_{\infty}\left(\alpha_{c}(f), \alpha_{c}(g)\right)<\varepsilon$ which proves that $\alpha_{c}$ is continuous too.

Following the same idea of proof one can give an analogous result for bounded Lipschitz mappings.

Theorem 4.5. Let $X$ be a $C B B(0)$ space, $Y$ a complete $C A T(0)$ space and $A \subseteq X$ nonempty. Then there exists a continuous mapping $\beta_{c}: \mathscr{L}(A, Y) \rightarrow \mathscr{L}(X, Y)$ such that for all $g \in \mathscr{L}(A, Y), \beta_{c}(g)(a)=g(a)$ for every $a \in A, \operatorname{Lip}\left(\beta_{c}(g), X\right)=\operatorname{Lip}(g, A)$ and $\beta_{c}(g)(X) \subseteq \overline{\operatorname{co}}(g(A))$.

In fact one can also consider the multivalued extension mappings:

- $\Phi_{c}: \mathscr{N}(A, Y) \rightarrow \mathcal{P}(\mathscr{N}(X, Y))$ which assigns to each nonexpansive mapping $f \in \mathscr{N}(A, Y)$ all its nonexpansive extensions $f^{\prime} \in \mathscr{N}(X, Y)$ with $f^{\prime}(X) \subseteq \overline{\mathrm{co}}(f(A))$.

- $\Psi_{c}: \mathscr{L}(A, Y) \rightarrow \mathcal{P}(\mathscr{L}(X, Y))$ which assigns to each Lipschitz mapping $f \in \mathscr{L}(A, Y)$ all its Lipschitz extensions $f^{\prime} \in \mathscr{L}(X, Y)$ with $\operatorname{Lip}(f, A)=\operatorname{Lip}\left(f^{\prime}, X\right)$ and $f^{\prime}(X) \subseteq \overline{\mathrm{co}}(f(A))$.

These mappings, too, will be lower semi-continuous.

Theorem 4.6. Let $\kappa \leq 0, X$ a $C B B(\kappa)$ space, $Y$ a complete $C A T(\kappa)$ space and $A \subseteq X$ nonempty. Then the mapping $\Phi_{c}: \mathscr{N}(A, Y) \rightarrow \mathcal{P}(\mathscr{N}(X, Y))$ is lower semi-continuous.

Proof. We show that for every $f \in \mathscr{N}(X, Y)$ with $f(X) \subseteq \overline{\mathrm{co}}(f(A))$ and for every $\varepsilon>0$ there exists $\delta>0$ such that every $g \in \mathscr{N}(A, Y)$ with $\sup _{a \in A} d_{Y}(f(a), g(a))<\delta$ admits an extension $g^{\prime} \in \mathscr{N}(X, Y)$ with $g^{\prime}(X) \subseteq \overline{\mathrm{co}}(g(A))$ and $d_{\infty}\left(f, g^{\prime}\right) \leq \varepsilon$.

Let $f$ be as above and $\varepsilon>0$. By Lemma 3.1 there exists $\delta>0$ such that every $g \in \mathscr{N}(A, Y)$ with $\sup _{a \in A} d_{Y}(f(a), g(a))<\delta$ admits an extension $g_{1} \in \mathscr{N}(X, Y)$ with $d_{\infty}\left(f, g_{1}\right) \leq \varepsilon / 3$.

Define $g^{\prime}: X \rightarrow Y, g^{\prime}(x)=P_{\overline{\mathrm{CO}}(g(A))}\left(g_{1}(x)\right)$. Clearly, $g^{\prime}$ is nonexpansive, extends $g$ and $g^{\prime}(X) \subseteq \overline{\mathrm{CO}}(g(A))$. Let $x \in X$. Then,

$$
d_{Y}\left(f(x), g^{\prime}(x)\right) \leq d_{Y}\left(f(x), g_{1}(x)\right)+d_{Y}\left(g_{1}(x), g^{\prime}(x)\right) \leq \varepsilon / 3+d_{Y}\left(g_{1}(x), g^{\prime}(x)\right) .
$$

For every $y \in \overline{\mathrm{co}}(g(A))$ we have that

$$
d_{Y}\left(g_{1}(x), g^{\prime}(x)\right) \leq d_{Y}\left(g_{1}(x), y\right) \leq d_{Y}\left(g_{1}(x), f(x)\right)+d_{Y}(f(x), y),
$$

from where

$$
d_{Y}\left(g_{1}(x), g^{\prime}(x)\right) \leq \varepsilon / 3+\operatorname{dist}(f(x), \overline{\operatorname{co}}(g(A))) .
$$

Consider $E=\{y \in Y: \operatorname{dist}(y, \overline{\mathrm{co}}(g(A))) \leq \varepsilon / 3\}$. We know that $f(A) \subseteq E$ since for any $a \in A$,

$$
\operatorname{dist}(f(a), \overline{\operatorname{co}}(g(A))) \leq d_{Y}(f(a), g(a)) \leq \varepsilon / 3 .
$$

Since $E$ is closed and convex we have that $\overline{\mathrm{co}}(f(A)) \subseteq E$. But $f(X) \subseteq \overline{\mathrm{co}}(f(A))$ and so $f(x) \in E$. Thus, dist $(f(x), \overline{\mathrm{co}}(g(A))) \leq \varepsilon / 3$ which implies that $d_{Y}\left(g_{1}(x), g^{\prime}(x)\right) \leq 2 \varepsilon / 3$. Using (4), we obtain that $d_{Y}\left(f(x), g^{\prime}(x)\right) \leq \varepsilon$.

The same argument yields the result for bounded Lipschitz mappings.

Theorem 4.7. Let $X$ be a $C B B(0)$ space, $Y$ a complete $C A T(0)$ space and $A \subseteq X$ nonempty. Then the mapping $\Psi_{c}: \mathscr{L}(A, Y) \rightarrow \mathcal{P}(\mathscr{L}(X, Y))$ is lower semi-continuous.

Note that one could apply, as in Section 3. Theorem 3.5 to the mappings $\Phi_{c}$ and $\Psi_{c}$ to obtain directly Theorems 4.4 and 4.5, respectively.

Remark 4.8. Similar results to the ones given in this section can be proved when $Y$ is a complete $\mathbb{R}$-tree and $X$ is a general metric space. 


\section{$5 \quad$ Nonexpansive selections in hyperconvex metric spaces}

We prove next that results for mappings $\Phi$ and $\Phi_{c}$ from previous sections can be strengthened if the target space is a hyperconvex metric space. Indeed, we will show that extensions of nonexpansive (Lipschitz) mappings can be chosen to be not only continuously but in a nonexpansive (or Lipschitz) way. Our result will follow as a direct application of the next result [10, Theorem 1] (see also [20, Theorem 1]). The class of externally hyperconvex subsets of a metric space $X$, defined in Section 2 , is denoted by $\mathscr{E}(X)$.

Theorem 5.1 (Khamsi, Kirk, Martínez-Yáñez [10]). Let $X$ be a metric space and $Y$ a hyperconvex metric space. If $T: X \rightarrow \mathscr{E}(Y)$ is a multivalued mapping, then there exists a selection mapping $f: X \rightarrow Y$ of $T$ such that $d(f(x), f(y)) \leq H(T(x), T(y))$.

This theorem implies in particular that if the multivalued mapping $T$ is nonexpansive (Lipschitz) then $f$ can be chosen nonexpansive (Lipschitz). The next fact we need is that the set $\mathscr{N}(X, Y)$ endowed with the supremum distance is hyperconvex. This is basically due to [10, Theorem 3] where the result is proved for $\mathscr{N}(Y, Y)$ with $Y$ hyperconvex, but the proof carries over with no modification to our case. We state the result as it is given in [10.

Theorem 5.2 (Khamsi, Kirk, Martínez-Yáñez [10]). Let $Y$ be hyperconvex and $\lambda>0$. Let $\lambda(Y, Y)$ be the family of all bounded $\lambda$-Lipschitz mappings from $Y$ into $Y$. Then $\lambda(Y, Y)$ is hyperconvex endowed with the supremum distance.

Next we show that the mapping $\Phi$ is nonexpansive.

Lemma 5.3. Let $X$ be a metric space, $A \subseteq X$ nonempty and $Y$ a hyperconvex metric space. Then $\Phi$ is a nonexpansive multivalued mapping.

Proof. Let $f, g \in \mathscr{N}(A, Y)$ and $f^{\prime} \in \Phi(f)$. We need to show that there exists $g^{\prime} \in \Phi(g)$ such that $d_{\infty}\left(f^{\prime}, g^{\prime}\right) \leq$ $d_{\infty}(f, g)$. We will construct $g^{\prime}$ point by point beginning with $g^{\prime}(a)=g(a)$ for all $a \in A$. Let $r=d_{\infty}(f, g)$, $x_{0} \in X \backslash A$ and consider the intersection

$$
A_{0}=\left(\bigcap_{a \in A} B\left(g^{\prime}(a), d\left(a, x_{0}\right)\right)\right) \bigcap B\left(f^{\prime}\left(x_{0}\right), r\right) .
$$

It is easy to see that these balls have nonempty intersection two-by-two and so, by hyperconvexity of $Y$, $A_{0} \neq \emptyset$. Define $g^{\prime}\left(x_{0}\right)$ as any element in $A_{0}$.

Let $x_{1} \in X \backslash\left(A \cup\left\{x_{0}\right\}\right)$ and consider now

$$
A_{1}=\left(\bigcap_{a \in A} B\left(g^{\prime}(a), d\left(a, x_{1}\right)\right)\right) \bigcap B\left(g^{\prime}\left(x_{0}\right), d\left(x_{0}, x_{1}\right)\right) \bigcap B\left(f^{\prime}\left(x_{1}\right), r\right) .
$$

Checking distances between centers, with $a, a_{1}, a_{2} \in A$, we have that:

$$
\begin{aligned}
d\left(g^{\prime}\left(a_{1}\right), g^{\prime}\left(a_{2}\right)\right) & \leq d\left(a_{1}, a_{2}\right) \leq d\left(a_{1}, x_{1}\right)+d\left(a_{2}, x_{1}\right), \\
d\left(g^{\prime}(a), g^{\prime}\left(x_{0}\right)\right) & \leq d\left(a, x_{0}\right) \leq d\left(a, x_{1}\right)+d\left(x_{0}, x_{1}\right), \\
d\left(g^{\prime}(a), f^{\prime}\left(x_{1}\right)\right) & \leq d\left(g^{\prime}(a), f^{\prime}(a)\right)+d\left(f^{\prime}(a), f^{\prime}\left(x_{1}\right)\right) \leq r+d\left(a, x_{1}\right), \\
d\left(g^{\prime}\left(x_{0}\right), f^{\prime}\left(x_{1}\right)\right) & \leq d\left(g^{\prime}\left(x_{0}\right), f^{\prime}\left(x_{0}\right)\right)+d\left(f^{\prime}\left(x_{0}\right), f^{\prime}\left(x_{1}\right)\right) \leq r+d\left(x_{0}, x_{1}\right) .
\end{aligned}
$$

Hence, by hyperconvexity of $Y$, we have that $A_{1}$ is nonempty. Choose $g^{\prime}\left(x_{1}\right)$ as any point in $A_{1}$. The proof is completed after a standard transfinite argument. We omit further details.

To be able to apply Theorem 5.1 we still need the values of $\Phi$ to be externally hyperconvex.

Lemma 5.4. Let $X$ be a metric space, $A \subseteq X$ nonempty and $Y$ a hyperconvex metric space. Then $\Phi(f)$ is externally hyperconvex in $\mathscr{N}(X, Y)$ for every $f \in \mathscr{N}(A, Y)$. 
Proof. We know that $\Phi(f)$ is nonempty due to Theorem 2.3. Let $f \in \mathscr{N}(A, Y),\left\{f_{\alpha}\right\}_{\alpha \in \mathscr{A}} \subseteq \mathscr{N}(X, Y)$ and $\left\{r_{\alpha}\right\}_{\alpha \in \mathscr{A}} \subseteq \mathbb{R}^{+}$such that $d_{\infty}\left(f_{\alpha}, f_{\beta}\right) \leq r_{\alpha}+r_{\beta}$ and $\operatorname{dist}\left(f_{\alpha}, \Phi(f)\right) \leq r_{\alpha}$ for all $\alpha, \beta \in \mathscr{A}$. We need to prove that

$$
\left(\bigcap_{\alpha \in \mathscr{A}} B\left(f_{\alpha}, r_{\alpha}\right)\right) \bigcap \Phi(f) \neq \emptyset
$$

We will construct an extension $f^{\prime}$ of $f$ in the above intersection by transfinite induction. Let $f^{\prime}(a)=f(a)$ for $a \in A$. Since $\operatorname{dist}\left(f_{\alpha}, \Phi(f)\right) \leq r_{\alpha}$ it is clear that $d\left(f_{\alpha}(a), f^{\prime}(a)\right) \leq r_{\alpha}$ for every $a \in A$. Let $x_{0} \in X \backslash A$ and consider the intersection

$$
A_{0}=\left(\bigcap_{a \in A} B\left(f^{\prime}(a), d\left(a, x_{0}\right)\right)\right) \bigcap\left(\bigcap_{\alpha \in \mathscr{A}} B\left(f_{\alpha}\left(x_{0}\right), r_{\alpha}\right)\right) .
$$

A two-by-two case study and the hyperconvexity of $Y$ (see the $A_{1}$ case below for more details) easily show that $A_{0}$ is nonempty. Define $f^{\prime}\left(x_{0}\right)$ as any point in $A_{0}$.

Taking now $x_{1} \in X \backslash\left(A \cup\left\{x_{0}\right\}\right)$, the corresponding intersection to look at is

$$
A_{1}=\left(\bigcap_{a \in A} B\left(f^{\prime}(a), d\left(a, x_{1}\right)\right)\right) \bigcap B\left(f^{\prime}\left(x_{0}\right), d\left(x_{0}, x_{1}\right)\right) \bigcap\left(\bigcap_{\alpha \in \mathscr{A}} B\left(f_{\alpha}\left(x_{1}\right), r_{\alpha}\right)\right) .
$$

We check next the hyperconvexity condition for $A_{1}\left(a, a_{1}, a_{2}\right.$ stand for points in $\left.A\right)$ :

$$
\begin{aligned}
d\left(f^{\prime}\left(a_{1}\right), f^{\prime}\left(a_{2}\right)\right) & \leq d\left(a_{1}, a_{2}\right) \leq d\left(a_{1}, x_{1}\right)+d\left(a_{2}, x_{1}\right), \\
d\left(f^{\prime}(a), f^{\prime}\left(x_{0}\right)\right) & \leq d\left(a, x_{0}\right) \leq d\left(a, x_{1}\right)+d\left(x_{0}, x_{1}\right), \\
d\left(f^{\prime}(a), f_{\alpha}\left(x_{1}\right)\right) & \leq d\left(f^{\prime}(a), f_{\alpha}(a)\right)+d\left(f_{\alpha}(a), f_{\alpha}\left(x_{1}\right)\right) \leq r_{\alpha}+d\left(a, x_{1}\right), \\
d\left(f^{\prime}\left(x_{0}\right), f_{\alpha}\left(x_{1}\right)\right) & \leq d\left(f^{\prime}\left(x_{0}\right), f_{\alpha}\left(x_{0}\right)\right)+d\left(f_{\alpha}\left(x_{0}\right), f_{\alpha}\left(x_{1}\right)\right) \leq r_{\alpha}+d\left(x_{0}, x_{1}\right), \\
d\left(f_{\alpha}\left(x_{1}\right), f_{\beta}\left(x_{1}\right)\right) & \leq r_{\alpha}+r_{\beta} .
\end{aligned}
$$

Therefore, $A_{1}$ is nonempty and it suffices to define $f^{\prime}\left(x_{1}\right)$ as any point in $A_{1}$. The proof is completed by transfinite induction.

Remark 5.5. Regarding the mapping $\Psi$, it does not seem that the approach applied to $\Phi$ in the hyperconvex case is also working.

Remark 5.6. Notice that Lemma[5.4 improves [20, Theorem 17].

Now, we can give the main result of this section.

Theorem 5.7. Let $X$ be a metric space, $A \subseteq X$ nonempty and $Y$ a hyperconvex metric space. Then there exists a nonexpansive mapping $\alpha: \mathscr{N}(A, Y) \rightarrow \mathscr{N}(X, Y)$ such that for all $g \in \mathscr{N}(A, Y), \alpha(g)(a)=g(a)$ for every $a \in A$.

Proof. It directly follows now from Lemmas 5.3 and 5.4 and Theorems 5.1 and 5.2 .

Remark 5.8. By considering adequate modifications in the proofs, Theorem 5.7 also holds for $\lambda(A, Y)$ instead of $\mathscr{N}(A, Y)$ and $\lambda$-Lipschitz extensions instead of nonexpansive extensions.

In particular, since complete $\mathbb{R}$-trees are hyperconvex spaces, we have the following corollary which improves the corresponding result from Section 3 (see Remark 3.8).

Corollary 5.9. Let $X$ be a metric space, $A \subseteq X$ nonempty and $Y$ a complete $\mathbb{R}$-tree. Then the multivalued mapping $\Phi$ admits a nonexpansive selection.

It is now natural to wonder about results from Section 4 in the hyperconvex setting. First, we need to clarify the notion of convex hull. A natural option in this case is to consider the admissible hull of a set. 
Definition 5.10. Let $X$ be a metric space and $A \subseteq X$ nonempty and bounded. Then the admissible hull $\operatorname{cov}(A)$ of $A$ is the intersection of all the closed balls containing $A$. A set is called admissible if it coincides with its admissible hull.

It is easy to see that

$$
\operatorname{cov}(A)=\bigcap_{x \in X} B\left(x, r_{x}(A)\right)
$$

where $r_{x}(A)=\sup \{d(x, a): a \in A\}$. Now we can define $\Phi_{c}$ as in Section廿4replacing $\overline{\operatorname{co}}(f(A))$ with $\operatorname{cov}(f(A))$. In order to prove Theorem 5.7 for $\Phi_{c}$ in the hyperconvex setting we only need to show that Lemmas 5.3 and 5.4 still hold true. This is indeed the case. We point out next how to modify the corresponding proofs.

Lemma 5.11. Let $X$ be a metric space, $A \subseteq X$ nonempty and $Y$ a hyperconvex metric space. Then $\Phi_{c}$ is a nonexpansive multivalued mapping.

Proof. First we need to show that $\Phi_{c}(f)$ is nonempty for $f \in \mathscr{N}(A, Y)$ which directly follows from the fact that admissible subsets of hyperconvex spaces are hyperconvex themselves and so, from Theorem 2.3 extensions $f^{\prime}$ of $f$ exist such that $f^{\prime} \in \mathscr{N}(X, \operatorname{cov}(f(A)))$. Now, define the set $A_{0}$ as in the proof of Lemma 5.3 in the following way:

$$
A_{0}=\left(\bigcap_{a \in A} B\left(g^{\prime}(a), d\left(a, x_{0}\right)\right)\right) \bigcap\left(\bigcap_{y \in Y} B\left(y, r_{y}(g(A))\right)\right) \bigcap B\left(f^{\prime}\left(x_{0}\right), r\right) .
$$

To apply hyperconvexity the only case which is not trivial is for pairs of balls centered at $y \in Y$ and at $f^{\prime}\left(x_{0}\right)$. But for this case we have that, since $f^{\prime} \in \mathscr{N}(X, \operatorname{cov}(f(A))), d\left(y, f^{\prime}\left(x_{0}\right)\right) \leq r_{y}(f(A))=\sup _{a \in A} d(y, f(a)) \leq$ $\sup _{a \in A}(d(y, g(a))+d(g(a), f(a))) \leq r_{y}(g(A))+r$. Therefore $A_{0} \neq \emptyset$ and we may define $g^{\prime}\left(x_{0}\right)$ as any point in $A_{0}$.

For the next step we need to consider

$$
A_{1}=A_{1}^{\prime} \cap B\left(g^{\prime}\left(x_{0}\right), d\left(x_{0}, x_{1}\right)\right),
$$

where

$$
A_{1}^{\prime}=\left(\bigcap_{a \in A} B\left(g^{\prime}(a), d\left(a, x_{1}\right)\right)\right) \bigcap\left(\bigcap_{y \in Y} B\left(y, r_{y}(g(A))\right)\right) \bigcap B\left(f^{\prime}\left(x_{1}\right), r\right) .
$$

Now, since $g^{\prime}(a) \in g(A)$ the case $d\left(g^{\prime}(a), y\right)$ for $a \in A$ and $y \in Y$ follows. The case $d\left(y, g^{\prime}\left(x_{0}\right)\right)$ follows by the previous step. The last case, $d\left(y, f^{\prime}\left(x_{1}\right)\right)$ follows just as the case discussed for $A_{0}$ and so $A_{1}$ is also nonempty. We complete the proof by transfinite induction.

The following lemma also holds.

Lemma 5.12. Let $X$ be a metric space, $A \subseteq X$ nonempty and $Y$ a hyperconvex metric space. Then $\Phi_{c}(f)$ is externally hyperconvex in $\mathscr{N}(X, Y)$ for every $f \in \mathscr{N}(A, Y)$.

Proof. This proof follows the same patterns as the one of Lemma 5.4. Let $f \in \mathscr{N}(A, Y),\left\{f_{\alpha}\right\}_{\alpha \in \mathscr{A}} \subseteq$ $\mathscr{N}(X, Y)$ and $\left(r_{\alpha}\right) \subseteq \mathbb{R}^{+}$such that $d_{\infty}\left(f_{\alpha}, f_{\beta}\right) \leq r_{\alpha}+r_{\beta}$ and $\operatorname{dist}\left(f_{\alpha}, \Phi_{c}(f)\right) \leq r_{\alpha}$ for all $\alpha, \beta \in \mathscr{A}$. We need to prove that

$$
\left(\bigcap_{\alpha \in \mathscr{A}} B\left(f_{\alpha}, r_{\alpha}\right)\right) \bigcap \Phi_{c}(f) \neq \emptyset \text {. }
$$

It is possible to construct an extension $f^{\prime}$ of $f$ in the above intersection by transfinite induction and this suffices to prove the lemma. Now the set $A_{0}$ to consider is given by:

$$
A_{0}=\left(\bigcap_{a \in A} B\left(f^{\prime}(a), d\left(a, x_{0}\right)\right)\right) \bigcap\left(\bigcap_{y \in Y} B\left(y, r_{y}(f(A))\right)\right) \bigcap\left(\bigcap_{\alpha \in \mathscr{A}} B\left(f_{\alpha}\left(x_{0}\right), r_{\alpha}\right)\right) .
$$


We need to check the hyperconvexity condition for $d\left(f^{\prime}(a), y\right)$ with $a \in A$ and $y \in Y$, and $d\left(f_{\alpha}\left(x_{0}\right), y\right)$ for $\alpha \in \mathscr{A}$ and $y \in Y$. The first case is trivial as $f^{\prime}(a)=f(a)$. For the second one, we need to recall that $\operatorname{dist}\left(f_{\alpha}, \Phi_{c}(f)\right) \leq r_{\alpha}$ and so, for $\varepsilon>0$, there exists $z\left(=z_{\alpha}\right) \in \operatorname{cov}(f(A))$ such that $d\left(f_{\alpha}\left(x_{0}\right), z\right) \leq r_{\alpha}+\varepsilon$. Therefore, for $y \in Y$ and $\alpha \in \mathscr{A}$,

$$
d\left(y, f_{\alpha}\left(x_{0}\right)\right) \leq d(y, z)+d\left(z, f_{\alpha}\left(x_{0}\right)\right) \leq r_{y}(f(A))+r_{\alpha}+\varepsilon .
$$

The hyperconvexity condition finally follows because $\varepsilon$ is arbitrary.

The set $A_{1}$ in this case is given by

$$
A_{1}=A_{1}^{\prime} \cap B\left(f^{\prime}\left(x_{0}\right), d\left(x_{0}, x_{1}\right)\right),
$$

where

$$
A_{1}^{\prime}=\left(\bigcap_{a \in A} B\left(f^{\prime}(a), d\left(a, x_{1}\right)\right)\right) \bigcap\left(\bigcap_{y \in Y} B\left(y, r_{y}(f(A))\right)\right) \bigcap\left(\bigcap_{\alpha \in \mathscr{A}} B\left(f_{\alpha}\left(x_{1}\right), r_{\alpha}\right)\right) .
$$

We only need to check intersections with balls centered at $y \in Y$. The cases $d\left(f^{\prime}(a), y\right)$ and $d\left(y, f_{\alpha}\left(x_{1}\right)\right)$ follow as above. The case that remains to check is $d\left(y, f^{\prime}\left(x_{0}\right)\right)$ which follows by construction and, in general, by the inductive hypothesis.

Finally, we can state the following result.

Theorem 5.13. Let $X$ be a metric space, $A \subseteq X$ nonempty and $Y$ a hyperconvex metric space. Then there exists a nonexpansive mapping $\alpha_{c}: \mathscr{N}(A, Y) \rightarrow \mathscr{N}(X, Y)$ such that for all $g \in \mathscr{N}(A, Y), \alpha_{c}(g)(a)=g(a)$ for every $a \in A$ and $\alpha_{c}(g)(X) \subseteq \operatorname{cov}(g(A))$.

Remark 5.14. Again, by considering adequate modifications in the proofs, one can see that Theorem 5.13 holds in fact for $\lambda(A, Y)$ and $\lambda$-Lipschitz extensions.

Remark 5.15. Theorem 1 in [20] gives the same result as Theorem[5.1 but for multivalued mappings with admissible values instead of externally hyperconvex subsets. As far as the authors know, Theorem 5.7 may be the first application of Theorem [5.1 where the externally hyperconvex condition plays a substantial role. In fact, values of the mapping $\Phi$, which have been proved to be externally hyperconvex, need not be admissible. Consider, for instance, $X$ as the real interval $[0,2], A \subseteq X$ as $[1,2]$ and $Y=[0,1]$. Define $f \in \mathscr{N}(A, Y)$ as the function constantly equal to 1 . Then the functions $g(x)=1$ for $x \in X$ and

$$
h(x)= \begin{cases}x & \text { if } x \in[0,1] \\ 1 & \text { if } x \in[1,2]\end{cases}
$$

are in $\Phi(f)$. Therefore, any ball in $\mathscr{N}(X, Y)$ containing $\Phi(f)$ must be of radius at least $1 / 2$ and, in particular, it must contain the function constantly equal to $3 / 4$ which is not in $\Phi(f)$.

Remark 5.16. In this section we approached the case $\Phi_{c}$ in a direct way and not going through the metric projection as in Section 母. In contrast to the case of $C A T(\kappa)$ spaces with $\kappa \leq 0$ where metric projections on closed and convex subsets are singlevalued, projections onto admissible subsets of hyperconvex metric spaces are multivalued. However, as it was shown in [20] they admit a nonexpansive selection (it was later shown in [10] that the same holds for externally hyperconvex subsets). This problem was further studied and the interested reader can find more about it in [6, [7].

\section{Acknowledgements}

Rafa Espínola was supported by DGES, Grant MTM2012-34847C02-01 and Junta de Andalucía, Grant FQM-127. Adriana Nicolae was supported by a grant of the Romanian Ministry of Education, CNCS UEFISCDI, project number PN-II-RU-PD-2012-3-0152. Part of this work was carried out while Adriana Nicolae was visiting the University of Seville. She would like to thank the Department of Mathematical Analysis and the Institute of Mathematics of the University of Seville (IMUS). 


\section{References}

[1] Alber, Y., Reich, S., Yao, J.-C.: Iterative methods for solving fixed-point problems with nonself-mappings in Banach spaces. Abstr. Appl. Anal. 2003, 193-216 (2003)

[2] Alexander, S., Kapovitch, V., Petrunin, A.: Alexandrov meets Kirszbraun. In: Akbulut, S., Auroux, D., Önder, T. (eds.) Proceedings of the Gökova Geometry-Topology Conference 2010, pp. 88-109, Int. Press, Somerville, MA (2011)

[3] Aronszajn, N., Panitchpakdi, P.: Extensions of uniformly continuous transformations and hyperconvex metric spaces. Pacific J. Math. 6, 405-439 (1956)

[4] Bridson, M.R., Haefliger, A.: Metric spaces of non-positive curvature. Springer, Berlin (1999)

[5] Burago, D., Burago, Y., Ivanov, S.: A course in metric geometry. In: Graduate Studies in Math., Vol. 33, Amer. Math. Soc., Providence, RI (2001)

[6] Espínola, R., Khamsi, M.A.: Introduction to hyperconvex spaces. In: Kirk, W.A., Sims, B. (eds.) Handbook of Metric Fixed Point Theory, pp. 391-435. Kluwer Academic Publishers, Dordrecht (2001)

[7] Espínola, R.: On selections of the metric projection and best proximity pairs in hyperconvex spaces. Ann. Univ. Mariae Curie-Skłodowska Sect. A 59, 9-17 (2005)

[8] Horvath, C.D.: Contractibility and generalized convexity. J. Math. Anal. Appl. 156, 341-357 (1991)

[9] Jost, J.: Nonpositive Curvature: Geometric and Analytic Aspects. In: Lect. in Math., ETH Zürich, Birkhäuser, Basel (1997)

[10] Khamsi, M.A., Kirk, W.A., Martínez-Yáñez, C.: Fixed point and selection theorems in hyperconvex spaces. Proc. Amer. Math. Soc. 128, 3275-3283 (2000)

[11] Kirk, W.A.: Hyperconvexity of $\mathbb{R}$-trees. Fund. Math. 156, 67-72 (1998)

[12] Kirszbraun, M.D.: Über die zusammenziehende und Lipschitzsche Transformationen. Fund. Math. 22, 77-108 (1934)

[13] Kopecká, E.: Extending Lipschitz mappings continuously. J. Appl. Anal. 18, 167-177 (2012)

[14] Kopecká, E.: Bootstrapping Kirszbraun's extension theorem. Fund. Math. 217, 13-18 (2012)

[15] Kopecká, E., Reich, S.: Continuous extension operators and convexity. Nonlinear Anal. 74, 6907-6910 (2011)

[16] Kuczumov, T., Stachura, A.: Extensions of nonexpansive mappings in the Hilbert ball with the hyperbolic metric I, II. Comment. Math. Univ. Carolin. 29, 399-410 (1988)

[17] Lang, U., Schroeder, V.: Kirszbraun's theorem and metric spaces of bounded curvature. Geom. Funct. Anal. 7, 535-560 (1997)

[18] Lang, U., Pavlović, B., Schroeder, V.: Extensions of Lipschitz maps into Hadamard spaces. Geom. Funct. Anal. 10, 1527-1553 (2000)

[19] Price, G.B.: The theory of integration. Trans. Amer. Math. Soc. 47, 1-50 (1940)

[20] Sine, R., Hyperconvexity and nonexpansive multifunctions. Trans. Amer. Math. Soc. 315, 755-767 (1989)

[21] Valentine, F.A.: Contractions in non-Euclidean spaces. Bull. Amer. Math. Soc. 50, 710-713 (1944)

[22] Valentine, F.A.: A Lipschitz condition preserving extension for a vector function. Amer. J. Math. 67, 83-93 (1945) 